\title{
Punctuated Evolution of the Electron Microscope
}

\author{
A. Howie, Cambridge
}

(Cavendish Laboratory, University of Cambridge)

\begin{abstract}
The 1986 Nobel Prize for Physics was shared equally between Ernst Ruska (see photo) for his pioneering work on the Electron Microscope and the team of Gerd Binnig and Heinrich Rohrer for their development of the Scanning Tunnelling Microscope a review of which by $A$. Baratoff we published in the November/December issue of last year.
\end{abstract}

Advances in instrumentation, so often a crucial factor in scientific discovery, are often only implicity recognised, particularly when it comes to the award of Nobel prizes. We prefer to celebrate the discovery of the antiproton rather than the construction of the machine which predictably rendered that discovery inevitable. Exceptions can be made when sufficiently novel principles such as stochastic cooling or phase contrast microscopy are involved in the design of the new instrument, but even then recognition depends not only on good performance but also on the exciting discoveries which follow. Engineers, builders and designers generally may be glad to be excused quite such stringent tests before receiving the highest accolades in their profession!

Microscopy is an old and fertile field for the flowering of the instrument maker's talents. Remarkable new developments such as scanning optical and scanning acoustic microscopy have captured attention in recent years. However, atomic resolution is a holy grail which has invested the invention of the scanning tunnelling microscope by Binnig and Rohrer, with a special excitement. How intriguing therefore that they should share the 1986 Nobel Prize for Physics with Ernst Ruska whose contributions to microscopy date with those of Zernike and indeed lie closer to the time of Abbe than to the present day! A study of the events leading to these two achievements reveals their many connections and contrasts as well as the great range of evolutionary tempo which the scientific edifice can tolerate and indeed requires.

Ruska's early work ${ }^{1}$ ) was done in the period $1929-32$ as a graduate student (mostly unpaid) in the team of Professor Max Knoll at the Technical University of Berlin, investigating the high speed cathode ray oscilloscope. Part of the background lay in the theory of the focussing action of a magnetic solenoid developed by Busch following some qualitative and imperfectly understood observations by Gabor of the concentrating effect of an iron-shrouded coil. A striking early achievement, testifying to Ruska's swift mastery of the formidable problems of practical electron optics at that time, was the quantitative verification of the theory - something which Busch himself had attempted but failed to accomplish. The crucial demonstration of a two-stage "microscope" with a magnification of about $\times 20$ using iron-free lens coils was achieved by Ruska in 1931.

Greatly improved magnetic pole-piece lenses designed by Ruska and his brother-in-law von Borries then led in 1934 to the operation of a two-stage instrument with a magnification of $\times 12000$ and a resolution of $50 \mathrm{~nm}$ comfortably ahead of the light microscope. At this point Ruska joined Siemens who were planning commercial production. This followed their acquisition of a patent that was the result of a rather opportunist application, based on purely conceptual proposals, made by their chief electrical engineer Reinhold Rudenberg. Subsequent development of the electron microscope swiftly became an international entreprise ${ }^{2}$ ) but its widespread use was hampered by difficulties in the preparation of specimens and the damage done to them by the vacuum or electron beam. Gabor's view was that although only cinders might remain, they could still be worth studying - an opinion regarded as too optimistic by the biologists. In fact, he was being unnecessarily gloomy since most of the scattering is elastic.

Like most present-day electron optics, Ruska's work was entirely based on the particle properties of the electron and, fortunately perhaps, he did not get to hear about its wave nature until 1932 . Diffraction effects in the specimen are, however, extremely important, particularly in the small angle range of scattering to which spherical aberration res-

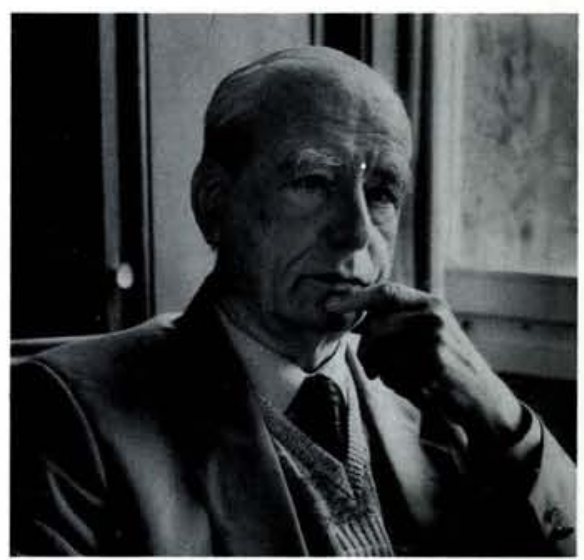

tricts the operation of electron lenses. The union between electron microscopy and electron diffraction, engineered by Boersch and LePoole proved of incalculable benefit to both disciplines ${ }^{3}$ ). Electron diffraction, previously plagued by problems of multiple scattering, found its true home in the interpretation of real space images. With the work of Hirsch and the Cambridge school, the diffraction contrast method of imaging crystal defects became the vehicle whereby the electron microscope replaced the $\mathrm{X}$-ray set as the primary structural tool of the materials scientist. In the past decade, with steady improvements in resolution to the $0.2 \mathrm{~nm}$ level directly revealing the atomic arrangement, structure imaging in the electron microscope has been enthusiastically adopted by biologists, mineralogists, chemists and most recently physicists with their interest in semiconductor interfaces and quantum well structures.

The high resolution transmission electron microscope of today still bears a remarkable resemblance to Ruska's original conception, making its development the most continuous and durable thread in the long history of electron optics. Scanning electron microscopy, using the same kind of lenses has emerged as an alternative tool, offering many advantages for the microanalysis and imaging of both specimen surfaces, although limited in resolution by source brightness problems. The solution to these, leading to Crewe's scanning transmission electron microscope with its ability to resolve and chemically identify individual atoms, has been provided by the tungsten field emission tip. The tungsten tip in the hands of Müller Crewe and most recently, Binnig and Rohrer, has now provided us with atomic images by three quite different routes and must surely rank with the magnetic lens as a key component of charged particle microscopy. It must be recognised though that the scanning 


\section{FREIE UNIVERSITÄT BERLIN \\ Professorship in Experimental Physics}

The Department of Physics at the Freie Universität Berlin announces an opening for a tenured full professor position in the field of experimental molecular physics. In addition to the regular teaching obligations the appointee is expected to build up a strong research activity within the Institute of Molecular Physics. In particular, this involves a leading engagement in the newly founded Sonderforschungsbereich "Energy and Charge Transfer in Molecular Aggregates", financed by the Deutsche Forschungsgemeinschaft. Accordingly, preference is given to candidates with a well-established record in physics of clusters or molecular aggregates.

Applicants should be experienced in teaching at the undergraduate and graduate level and should have internationally recognized research merits in molecular physics.

To apply, please send curriculum vitae, list of publications, teaching records, and names for references to the:

Department Head, Fachbereich Physik, Freie Universität Berlin,

Arnimallee 14, D-1000 Berlin

tunnelling microscope offers such tremendous advantages in terms of beam damage, range of operating pressure and ability to probe valence states that it may well displace the conventional electron microscope for surface imaging work. If so, this will represent a return of microscopists to an instrumental concept reminiscent of Leeuwenhoek in its simplicity.

\section{REFERENCES}

1. Ruska E., The Early Development of Electron Lenses and Electron Microscopy (Hirzel Verlag, Stuttgart) 1980.

2. Hawkes P.W. (ed.) "The Beginnings of Electron Microscopy", Advances in Electronics and Electron Physics Suppl. 16 (Academic Press) 1985.

3. Goodman P. (ed.) Fifty Years of Electron Diffraction (Riedel, Dordrecht) 1981.

\section{ISTITUTO DI FISICA NUCLEARE Post-Doctoral Fellowships in Experimental Physics}

Two in accelerator physics (for non-Italians only) valid for one year at the INFN Laboratori Nazionali di Frascati. Applications are invited from accelerator physicists to participate in the experimental programmes on the $350 \mathrm{MeV} \mathrm{e} \pm$ Linac and $1.5 \mathrm{GeV}$ storage ring ADONE and in the design of new facilities (s.c. RF cavities, single pass FEL experiments).

Two in nuclear structure physics (open to non-Italians) valid for one year from 1 Oct. 1987 to initiate and assist with research on the XTU tandem van de Graaff at the National Laboratories of Legnaro. Applications are invited from physicists holding a Ph.D. degree in nuclear structure physics.

Two in nuclear physics (for non-Italians only) valid for one year starting November 1987, to undertake research at the National Southern Laboratory in Catania and assist in the preparation of experiments and the machine development programmes.

Salary is LIT 24 million gross per annum = LIT 1600000 net, plus travel. Applications with curriculum vitae, publications list and three letters of reference to respectively:

Prof. Sergio Tazzari, Director, Laboratori Nazionali di Frascati, Via E. Fermi 40, I-00044 Frascati (Roma)

Prof. Pietro Dalpiaz, Director, Lab. Nazionali di Legnaro dell'INFN, Via Romea 4, 1-35020 Legnaro (Padova)

Prof. Nicola Cabibbo, President, National Inst. of Nuclear Physics, Casella Postale 56, I-00044 Frascati (Roma)

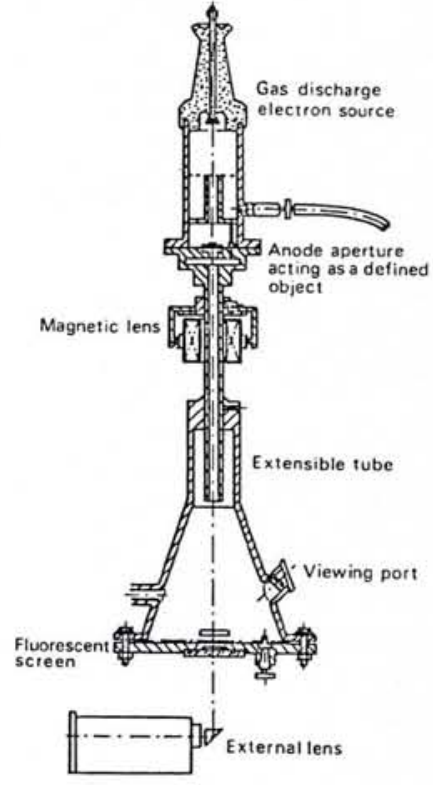

Above - Ruska's apparatus for investigating the focal properties of a magnetic lens (1929).

Below - A modern electron microscope, the JEM-2000 FX of JEOL Ltd.

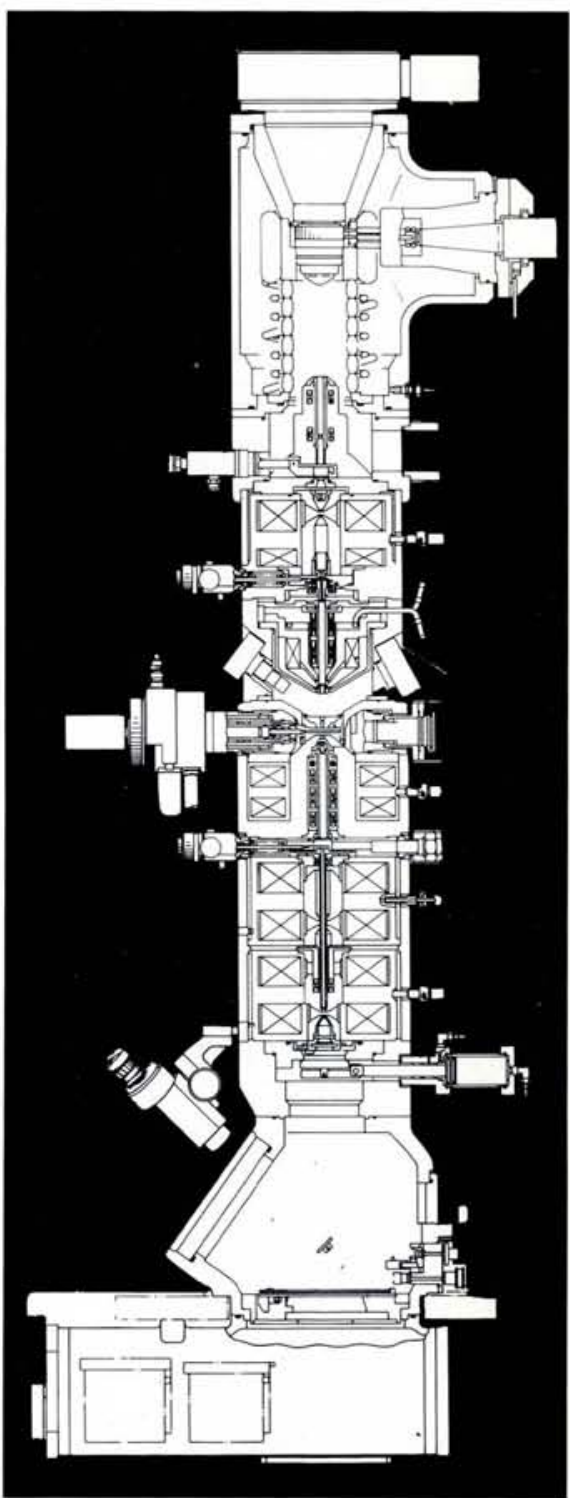

